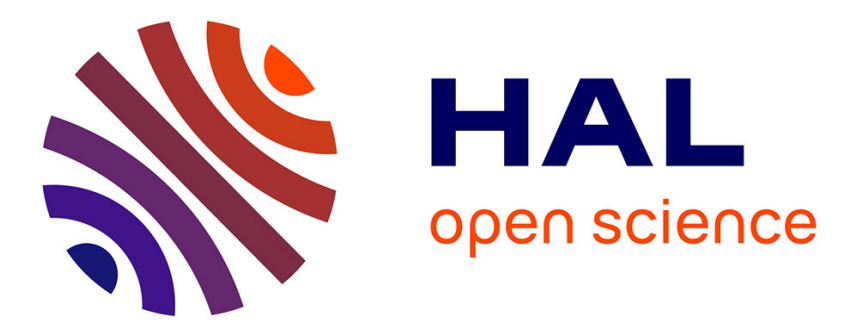

\title{
The capillary interaction between pairs of granular rafts
}

Antoine Lagarde, Christophe Josserand, Suzie Protiere

\section{To cite this version:}

Antoine Lagarde, Christophe Josserand, Suzie Protiere. The capillary interaction between pairs of granular rafts. Soft Matter, 2019, 10.1039/c9sm00476a . hal-02172301

\section{HAL Id: hal-02172301 \\ https://hal.science/hal-02172301}

Submitted on 17 Nov 2020

HAL is a multi-disciplinary open access archive for the deposit and dissemination of scientific research documents, whether they are published or not. The documents may come from teaching and research institutions in France or abroad, or from public or private research centers.
L'archive ouverte pluridisciplinaire HAL, est destinée au dépôt et à la diffusion de documents scientifiques de niveau recherche, publiés ou non, émanant des établissements d'enseignement et de recherche français ou étrangers, des laboratoires publics ou privés. 


\title{
Journal Name
}

\section{ARTICLE TYPE}

Cite this: DOI: $10.1039 / x x x x x x x x x x$

\section{The capillary interaction between pairs of granular rafts $^{\dagger}$}

\author{
Lagarde Antoine ${ }^{a}{ }^{\text {Josserand Christophe },{ }^{b} \text { and Protière Suzie }}{ }^{* a}$
}

Received Date

Accepted Date

DOI: 10.1039/xxxxxxxxxx

www.rsc.org/journalname

\begin{abstract}
When an object is placed at the surface of a liquid, its weight deforms the interface. For two identical spherical objects, such a deformation creates an attractive force, leading to the aggregation of the two-body system. Here, we experimentally investigate the interaction between two granular rafts, formed by the aggregation of dense millimeter-sized beads placed at an oil-water interface. The interfacial deformation created by such a two-dimensional object exceeds by at least an order of magnitude the deformation of a single bead. This leads to unusually high capillary forces which strongly depend on the number of particles. Likewise, because the raft grows in size as more particles are added, the viscous drag experienced increases along with the capillary attraction, leading to a non-trivial dependence of the balance of forces on the number of beads. By studying the relative motion of two granular rafts in relation with the interfacial deformation they generate, we derive a model for the observed speed profiles. With this work, we generalize how the capillary interaction between two non-identical complex structures evolves with their respective geometry.
\end{abstract}

\section{Introduction}

When a few identical objects are deposited on a fluid interface, a daily yet striking phenomenon occurs, during which the passive objects will spontaneously move towards one another. The implications of what could appear as a trivial experiment are countless. In Nature, fire ants use it to survive flood by aggregating into a floating raft ${ }^{1}$, while some animals are able to propel by deforming the water surface in a specific way 2 . This phenomenon is also used in industrial processes for the fabrication of macroscopic objects with a specific microstructure 3 3.5.

The self-assembly of objects at a liquid interface relies on the interaction due to the surface deformation around each particle ${ }^{6}$. When the menisci around several objects overlap, capillary attraction (or repulsion) arises. Colloidal particles generate lateral forces due to the undulation of the contact line around their surface $\frac{7-10}{10}$, either because of surface roughness and wettability, or due to a non-spherical shape. The interface around the particle is then conveniently seen as a capillary multipole. For anisotropic colloids, the interaction depends strongly on the individual shapes ${ }^{11}$, giving rise to oriented aggregation either through a precise manufacturing of the form of each particle $\frac{12}{12}$, or thanks to a curved interface acting as a force field 13 .

For larger objects, another contribution comes from the parti-

\footnotetext{
${ }^{a}$ Sorbonne Université, Centre National de la Recherche Scientifique, UMR 7190, Institut Jean Le Rond J'Alembert, F-75005 Paris, France.E-mail: protiere@ida.upmc.fr

${ }^{b}$ LadHyX, CNRS, Ecole Polytechnique, UMR 7646, 91128 Palaiseau, France.

$\dagger$ Electronic Supplementary Information (ESI) available: [details of any supplementary information available should be included here]. See DOI: 10.1039/b000000x/
}

cles' weight which deforms the surrounding interface by pushing the liquid down, an effect that can be taken into account through a generalized Archimedes' principle $\frac{14}{}$. Models for such interactions have been developed almost exclusively for two identical rigid bodies $\frac{15[16}{}$. In 1949, Nicolson ${ }^{15}$ proposed a model using a linear superposition approximation of the interface deformations to describe the force of interaction between two floating bubbles at a liquid interface. This far-field calculation leads to an analytical formulation of the capillary force between identical objects (bubbles, solid sphere, ...) 16/17. Good experimental agreement has been reported for the relative motion of a small number of large identical spheres $\frac{18[19}{19}$. However, most of these models and experiments concern individual and identical particles and no detailed investigations have been made so far to characterize the interactions between clusters of particles.

In this paper, we focus on such large objects, where dense millimeter-sized beads act as monopoles. We study the interaction between two granular rafts 2021 , each formed by the aggregation of dense spherical beads into a single axisymmetric object. Such 2-D structures are flexible and exhibit high deformations of the interface due both to their weight and their geometrical extension. The overall meniscus created by these large objects generates a specific raft-raft interaction that needs to be characterized. We quantify the dependence of the drag and the capillary force experienced by a raft on both its size and the size of the other interacting raft. The present study questions whether two assemblies of particles forming dense objects can interact as single entities, and investigates the influence of the clusters di- 
mensions on their interactions.

\section{Kinematics of two interacting granular rafts}

\subsection{Experimental setup}

In a typical experiment, a thick layer of oil (kinematic viscosity $v=50.10^{-6} \mathrm{~m}^{2} \cdot \mathrm{s}^{-1}$, density $\rho_{o}=960 \mathrm{~kg} \cdot \mathrm{m}^{-3}$, oil-water surface tension $\gamma=38 \mathrm{mN} . \mathrm{m}^{-1}$ ) is carefully poured into a tank of dimensions $0.2 \times 0.2 \times 0.25 \mathrm{~m}$ filled with pure water. A precise number of particles $n_{A}$ are sprinkled from above. Beads are deposited far away from one another, from every direction around the raft. Due to the very strong gravitational interaction between such objects the beads automatically aggregate into a compact axisymmetric raft. When using the beads presented in table 1 , we never observe a loosely packed assembly such as the one described in $22 \mid 23$. The raft size can be controlled by adding beads progressively (meaning the raft is made of precisely $n_{A}$ particles), as represented in figure 1

The same procedure is reproduced elsewhere in the tank, with $n_{B}$ particles. The motion of the two rafts thus formed (respectively identified by the letters A and B) is recorded either from above or from the side by a camera at 250 frames per second. The properties of the various beads used in the experiments are summarized in table 1, along with the approximate maximum number of particles $n_{\max }$ the corresponding raft can reach before sinking 21 .

Table 1 Characteristics of the beads used in experiments

\begin{tabular}{llll}
\hline Type of particle & $\rho_{s}\left(\mathrm{~kg} \cdot \mathrm{m}^{-3}\right)$ & $R_{\text {part }}(\mathrm{mm})$ & $n_{\max }$ \\
\hline Plastic & 1,420 & 2 & 16 \\
Plastic & 1,420 & 2.5 & 7 \\
Ceramic & 3,800 & 0.35 & 160 \\
Ceramic & 3,800 & 0.45 & 60 \\
Ceramic & 4,800 & 0.35 & 60 \\
Ceramic & 4,800 & 0.45 & 25 \\
\hline
\end{tabular}

At the oil-water interface, the capillary length $\ell_{c}=$ $\sqrt{\gamma /\left(\left(\rho_{w}-\rho_{o}\right) g\right)}$ is greatly increased by buoyancy effects, so that $\ell_{c} \approx 10 \mathrm{~mm}$ (with $\rho_{w}$ the density of water, and $g$ the gravitational acceleration). The maximal possible deformation is thus far more important than at an air-water interface. This leads to unusually high long-range capillary forces.

\subsection{Speed measurement}

For a given set of sizes $\left(n_{A}, n_{B}\right)$, we record the dynamics from above to measure the speed of the rafts, and from the side when we want to access the deformation of the interface. A typical experiment can be visualized in figure 2 (see supplementary information for the movies, taken from above and from the side), for two rafts with respectively $n_{A}=30$ and $n_{B}=50$. The two rafts move towards one another until they come into contact, at which point they will rearrange to form a bigger raft or sink. Here, we focus on the interaction of two rafts before they reach contact.

In a typical experiment such as the one presented in figure 2 a, the fluid interface undergoes strong deformations due to the weight of each raft. This induces a vertical displacement which cannot be neglected in our measurement of the raft speed. For example, in figure 2 a, the amplitude of the vertical motion of raft $\mathrm{B}\left(n_{B}=30\right)$ between the last two images is more than half the length of its horizontal motion. In our study, all the speed measurements are derived from top views of the granular raft motion. Yet, a movie taken from above only gives us information on the horizontal projection of the speed. The vertical component is not directly accessible. We deduce the vertical displacement from the radial motion, via measurements of the interface deformation.

The equation for the interface is obtained by a classic equilibrium between the hydrostatic pressure and the pressure jump due to the curvature. For a cylindrical coordinate system centered in the middle of the raft, the height of the interface beyond the raft $\mathrm{B}$ is obtained as the solution of the following system of equations:

$$
\left\{\begin{array}{l}
\gamma\left(\frac{h^{\prime \prime}}{1+h^{\prime 2}}+\frac{h^{\prime}}{r}\right)-\left(\rho_{w}-\rho_{o}\right) g h \sqrt{1+h^{\prime 2}}=0 \\
h(r \rightarrow \infty)=0 \\
h\left(r=R_{\text {raft B }}\right)=h_{\text {raft B }}
\end{array}\right.
$$

with $h$ the height of the interface ( $h=0$ for a flat interface), $r$ the radial coordinate, $h^{\prime}$ the derivative of $h$ with respect to $r, R_{\text {raft } B}$ the radius of the raft and $h_{\text {raft } B}$ the height of the interface at the edge of the raft (see figure 1). We neglect the irregularities at the edge of the raft due to the presence of the particles, making the assumption that the shape of the interface around the raft is isotropic. We can thus write equation (1) in an axisymmetric configuration.

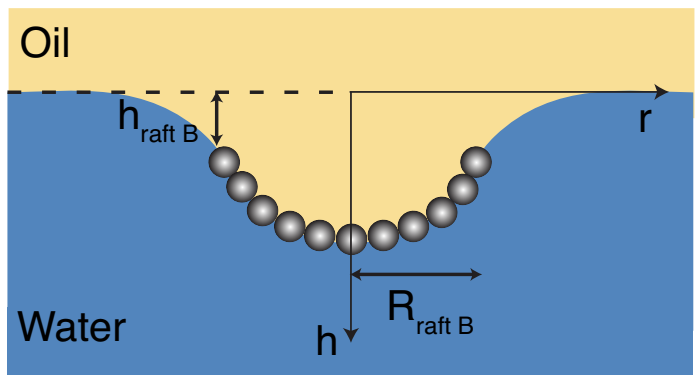

Fig. 1 Schematic side view of a granular raft. Deformation of the interface around a single raft $B$, described by two quantities: the radius of the raft $R_{\text {raft } B}$, and the depth of the interface at the edge of the raft $h_{\text {raft } B}$. The radial coordinates are defined from the center of the raft, while the origin of the vertical axis $h$ corresponds to the height of the undisturbed flat interface.

After having measured $R_{\text {raft } B}$ and $h_{\text {raft } B}$ from a side view of raft $B$ (without the presence of A), we solve equation (1) numerically, and obtain the expression of $h(r)$ imposed by the presence of the raft $\mathrm{B}$. Then, from the knowledge of the radial position $L$ of the center of $A$ with respect to the center of $B$, which is measured directly from the movie, we can deduce the expected vertical position of the granular raft A along its motion. Following this procedure, we can calculate the total speed $V$ of a granular raft from its radial displacement. Implicitly here, we use the Nicolson linear approximation ${ }^{15}$ by saying that the derivative of the vertical position of A only depends on the interface height imposed by $\mathrm{B}$.

The total speed $V$ for the experiment of figure 2 a is plot- 

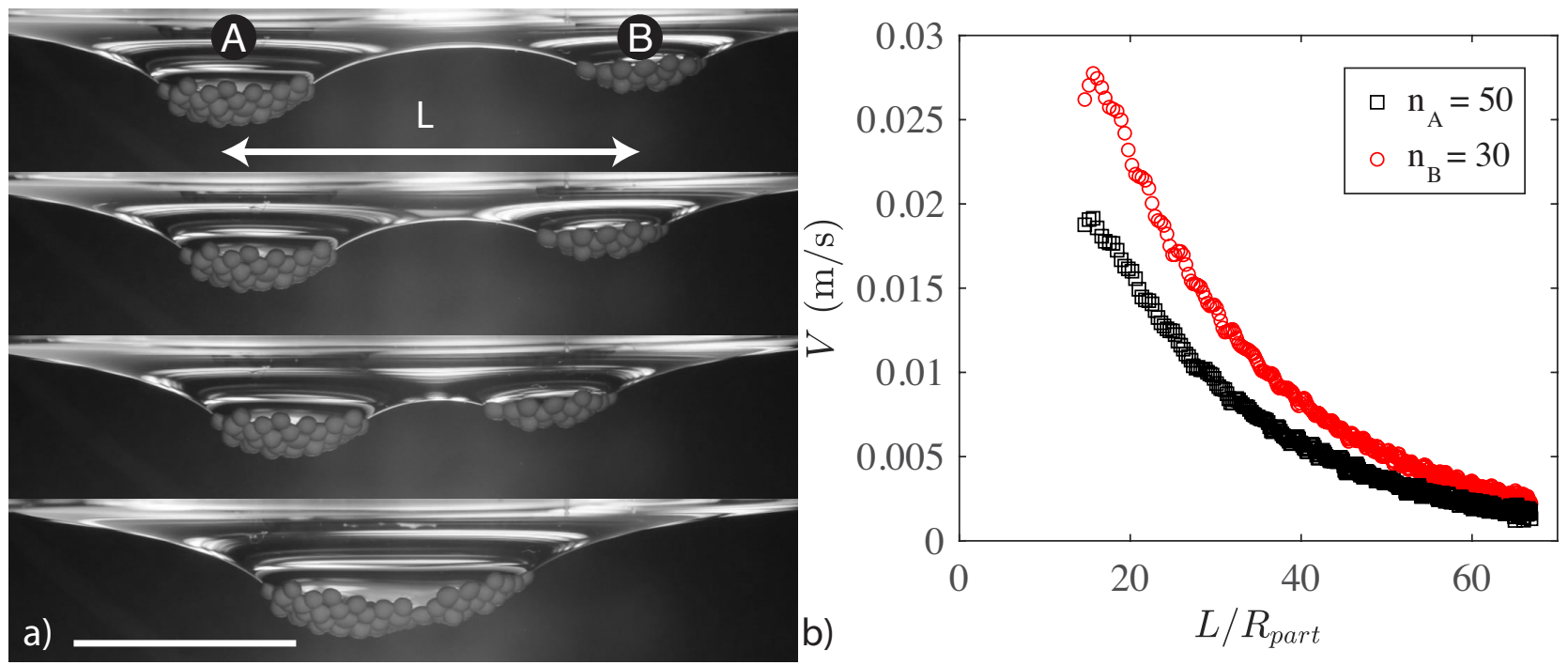

Fig. 2 Aggregation of two granular rafts. a) Time lapse of the motion of two granular rafts at an oil-water interface, filmed from the side (see supplementary information for the movies, taken from above and from the side). Each raft is made of ceramic spherical beads (density $\rho_{s}=3,800 \mathrm{~kg} \cdot \mathrm{m}^{-3}$, radius of a single particle $R_{\text {part }}=0.45 \mathrm{~mm}$ ), in an axisymmetric configuration, with 50 particles for the raft on the left (raft A), and 30 particles for the one on the right (raft B). The final image corresponds to the exact moment of contact between the two rafts. Time between two images: $0.13 \mathrm{~s}$. Scale bar: $10 \mathrm{~mm}$. b) Speed of the same two rafts as a function of the distance between the centers of the two rafts (made dimensionless using the radius of a bead $\left.R_{\text {part }}\right)$. Red circles: speed of raft B; black squares: speed of raft A. The errors bars are of the order of the thickness of the curves.

ted in figure $2 \mathrm{~b}$, for the two rafts, as a function of the nondimensionalized distance between the two centers. As a consequence, the curves have to be read from right to left, with an increase of $V$ as the two rafts get closer, until it reaches a maximum speed. Then, the two rafts briefly slow down just before contact, because of a hydrodynamic coupling in the drag force: the liquid that separates the two rafts before collision has to be expelled, causing an increase in the drag.

Qualitatively, we can already see in figure 2 that the sizes of the two rafts have an effect on their motion. Indeed a raft made of 30 particles will move at a larger speed than the raft made of 50 particles during their interaction. We now need a model to relate these speed profiles to the interacting forces acting on a granular raft.

\section{Dynamics of two interacting granular rafts}

\subsection{Theoretical model}

We study the influence of the number of particles on the respective motion of two granular rafts. To that end, we use the theoretical framework derived for the dynamics of two identical spheres interacting at an interface, in the limit of small deformations ${ }^{16}$, and see how the amplitude of the forces is affected by the size of the raft.

The force between two objects at an interface depends only on the shape of the interface around them, and is obtained by integrating the capillary forces around their contact line. This shape results from how the particles deform the interface, which in the case presented here is the consequence of the combined effect of their weight, their wettability and the induced buoyancy. In the case of small spheres $\left(R_{\text {part }} \ll \ell_{c}\right)$ where the shape of the interface is isotropic around each bead and its deformations are small, the calculation leads to an energy of interaction equal to the prod- uct between the effective weight of one particle and the vertical deformation created by the other. For two identical spheres, the force exerted by one bead on the other is then obtained by deriving the interaction energy, leading to:

$$
F_{\text {cap } 1 \rightarrow 1}=a\left(R_{\text {part }}, \rho_{s}, \ldots\right) K_{1}\left(\frac{L}{\ell_{c}}\right)
$$

with $a$ a coefficient taking into account the particle and fluid properties ( 16 for more details), $L$ the distance between the centers of the two particles, and $K_{1}$ the modified Bessel function of the second kind of order one. We generalize this formulation to the attraction of two granular rafts (designated by the letters A and B), as schematically represented in figure 3 ,

$$
F_{\text {cap } A \rightarrow B}=f\left(n_{A}, n_{B}\right) a\left(R_{\text {part }}, \rho_{s}, \ldots\right) K_{1}\left(\frac{L}{\ell_{c}}\right)
$$

where $f$ is the function we want to determine experimentally, $F_{\text {cap } A \rightarrow B}$ the force exerted by the raft A on B, and $n_{A}$ and $n_{B}$ the number of particles in each raft. To make such a generalization, we assume here that the wavelength and amplitude of the undulation of the edge of the raft are small enough so that they can be neglected at long range and the interface around the raft can be described as isotropic. We neglect here the granular nature of the raft, the edge of which may be roughened by individual particles.

The drag force acting on a raft also needs a careful treatment. For two identical spherical beads, $F_{\text {drag }}$ is expressed as a Stokes drag corrected by the mobility function $G$ for two spheres in a single phase, in order to take into account the drainage of the liquid between the particles:

$$
F_{\text {drag }}=b V G^{-1}\left(\frac{L}{R_{\text {part }}}\right)
$$




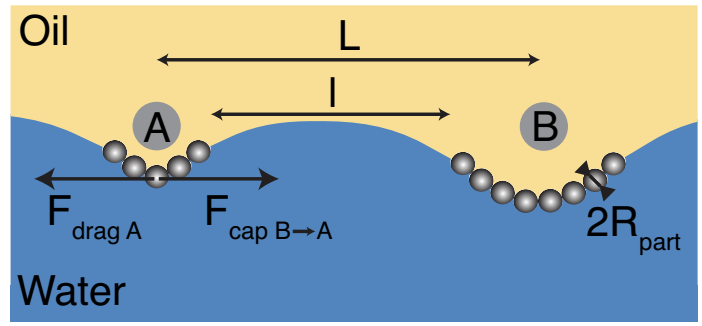

Fig. 3 Forces acting on a given axisymmetric granular raft. Schematic representation of the interaction between two granular rafts $\mathrm{A}$ and $\mathrm{B}\left(n_{A}\right.$ and $n_{B}$ respectively stand for the number of particles in each raft), at an oil-water interface. $L$ is the distance between the centers of the two rafts, while $l$ is the distance between the two nearest particles of each raft. The raft $A$ (respectively $B$ ) is subjected to a capillary attraction $F_{\text {cap } B \rightarrow A}$ and a viscous drag $F_{d r a g} A$ (respectively $F_{\text {cap } A \rightarrow B}$ and $F_{d r a g} B$ ).

with $V$ the speed of the particle, $b=6 \pi \mu_{o} R_{\text {part }} k$ a corrected Stokes drag coefficient on a sphere, where $k$ accounts for the fact that the particle moves along an interface and as a consequence is immersed in two phases 24 . The mobility function $G$ was tabulated by Batchelor ${ }^{25}$, and can be approximated by an interpolation formula 18 . At infinity, there is no coupling and $G(+\infty)=1$, whereas when the two particles are in contact, $G(2)=0$.

Again, we generalize equation (4) to the motion of a granular raft:

$$
F_{\text {drag } A}=g\left(n_{A}\right) b V_{A} G^{-1}\left(\frac{l+2 R_{\text {part }}}{R_{\text {part }}}\right)
$$

where $g$ is the function we want to determine experimentally, and $l$ designates the distance between the edges of the two closest particles of the two rafts, as defined in figure 3 . We approximate the hydrodynamic coupling between the two rafts by considering the drainage of the liquid only between the two closest particles of each interacting raft. Such a formulation of the drag is only valid for a sphere, but here we use it for a granular raft, which has the shape of a curved disk. As a consequence, the scaling law for the drag should also differ from the Stokes drag of a sphere, although we expect the general scaling laws for Stokes drag to be valid.

Keeping in mind the assumption lying under the scaling law of equation (5), we combine it with equation (3) and finally deduce an expression for the speed of a raft made of $n_{A}$ particles attracted by a second raft constituted by $n_{B}$ beads:

$$
V_{A}=\frac{f\left(n_{A}, n_{B}\right)}{g\left(n_{A}\right)} \frac{a}{b} G\left(\frac{l+2 R_{\text {part }}}{R_{\text {part }}}\right) K_{1}\left(\frac{L}{\ell_{c}}\right)
$$

The ratio $a / b$ is measured once and for all for each type of particle (given radius and given density, see table 1) thanks to a two-bead experiment, for which $f(1,1)=g(1)=1$ by definition. Then, $f / g$ can be experimentally determined as a function of $n_{A}$ and $n_{B}$, giving us information on the ratio of the two forces.

\subsection{Experimental results}

We plot in figure 4 the speed of the raft $\mathrm{A}$ as a function of the distance between the centers of the two rafts $L$, first with $n_{A}=1$ and an increasing $n_{B}$, and then with $n_{B}=60$ and an in- creasing $n_{A}$. The speed of the raft appears to increase both with $n_{A}$ and $n_{B}$, as expected from our definition of the capillary force. This is due to the deformation of the interface that increases with $n_{B}$. Similarly, $F_{\text {cap } B \rightarrow A}$ is related to the weight and the size of the raft $\mathrm{A}$, an increasing quantity with $n_{A}$. Focusing on a single curve of figure 4, we recover the behavior described previously: an increasing speed as the two rafts are attracted towards one another, until a maximum where the speed then starts to decrease due to the drainage of the liquid between the two rafts. As $n_{A}$ or $n_{B}$ increases, the curves are shifted to the right since $L_{\text {min }}=R_{\text {raft } A}+R_{\text {raft } B}$, a value which increases as we add particles.

The black dotted curves of figure 4 represent the fit of equation (6) for each couple of rafts $\left(n_{A}, n_{B}\right)$. There is a good agreement between the experimental data and the theory for $n_{A}=1$ (figure 4 a), the fitting line being within the experimental noise. As we increase $n_{A}$, the fit starts to drift from the measured speed, in particular regarding the position of its maximum. We discuss the limitations of our description in the last section of the paper and propose a number of hypothesis which may explain this result. Keeping in mind that for high $n_{A}$, our model does not explain the entire dynamics, we observe the evolution of the fitting coefficient $f / g$ as a function of $n_{A}$ and $n_{B}$ for different types of beads (see table 1), and a large variety of combinations of $n_{A}$ and $n_{B}$ (figure 5). All the data collapse onto the same straight line when represented as a function of $n_{B} \sqrt{n_{A}}$, on approximately three decades, giving the following experimental result:

$$
\frac{f\left(n_{A}, n_{B}\right)}{g\left(n_{A}\right)}=n_{B} \sqrt{n_{A}}
$$

Equation (7) gives us an empirical relation between the ratio of the two forces and the number of particles in each raft. Even though both forces are functions of $n_{A}, n_{B}$ only intervenes in the capillary force. This immediately proves that $F_{\text {cap } A \rightarrow B} \propto n_{B}$. Moreover, applying Newton's third law 26 , we obtain that this force has to be symmetrical in $n_{A}$ and $n_{B}$ and thus $F_{\text {cap } A \rightarrow B} \propto$ $n_{A} n_{B}$, implying also for the drag force $g\left(n_{A}\right) \propto \sqrt{n_{A}}$. The far-field behavior of the capillary force $F_{\text {cap } A \rightarrow B} \propto n_{A} n_{B}$ follows a Coulomb or gravitational-like law, replacing the electric charge or the mass by the number of grains; since this force only depends on the number of grains, it indicates that the organization of the particles within such compact granular rafts plays no role in the interaction. This is consistent with the superposition approximation, where interface deformation at large distances can be considered as the sum of the individual meniscii of each particle. However we lack a physical understanding of these scalings and need to go back to the definition of the capillary force.

\section{The drag and the capillary force}

As explained earlier, the capillary force exerted by a raft, assuming it has an isotropic shape and the deformations of the interface are small, is proportional to the slope of the interface deformed by its presence. In the limit of small deformations we derive an analytical formula for this slope by solving a linearized 

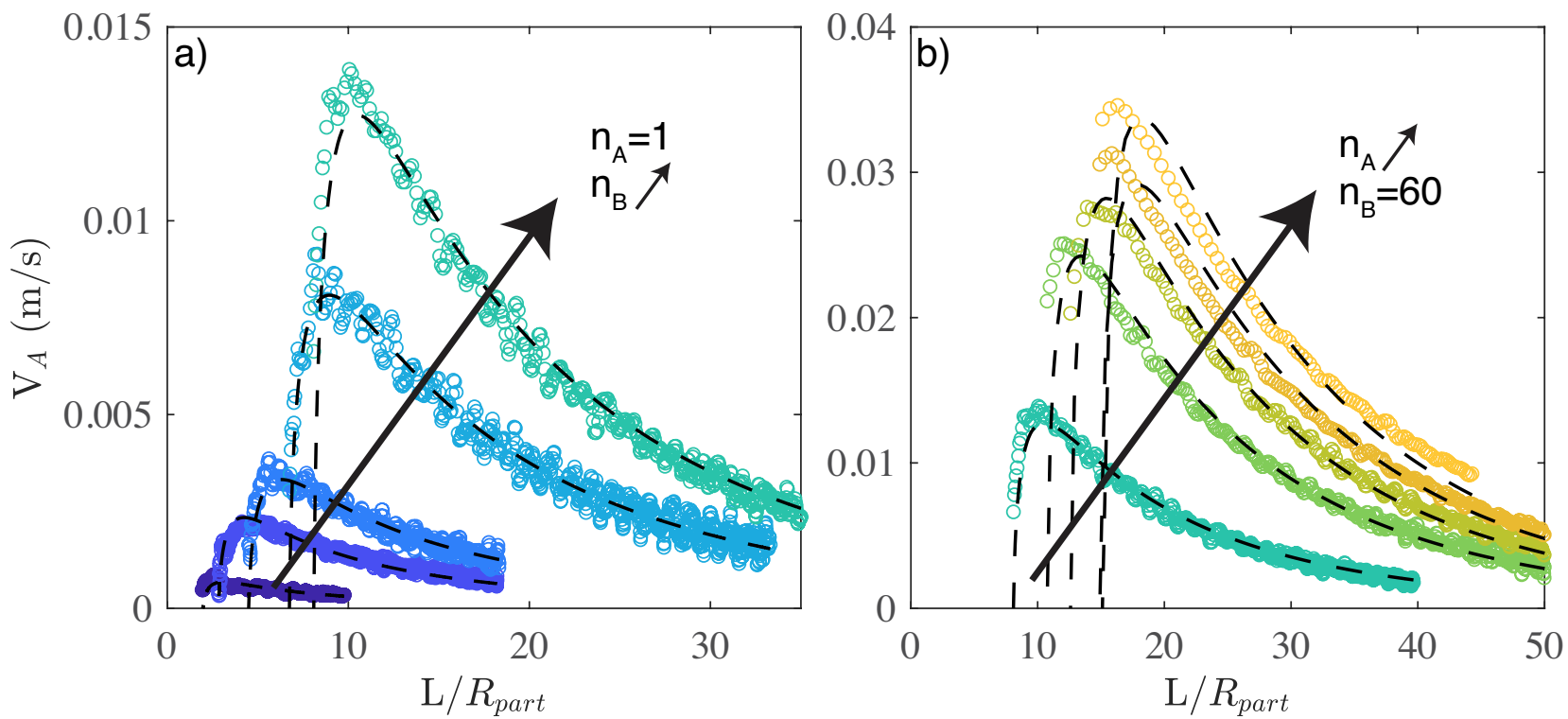

Fig. 4 Agreement between experimental results and theoretical model. Speed of the raft A for different number of particles in A and B, as a function of the distance between the centers of the two rafts (made dimensionless using the radius of a bead $R_{\text {part }}$ ), fitted by the theoretical prediction of equation 6 (black dotted curves). For all these experiments, the particles are ceramic spherical beads (density $\rho_{s}=3,800 \mathrm{~kg} \cdot \mathrm{m}^{-3}$, radius of a single particle $R_{\text {part }}=0.45 \mathrm{~mm}$ ). a) Speed of a single particle A for different sizes of the other raft, $n_{B}=1,5,10,30,60$. b) Speed of a raft of increasing size $\left(n_{A}=1,5,10,20,40\right)$, attracted by a raft of fixed size $\left(n_{B}=60\right)$. The errors bars are of the order of the thickness of the curves.

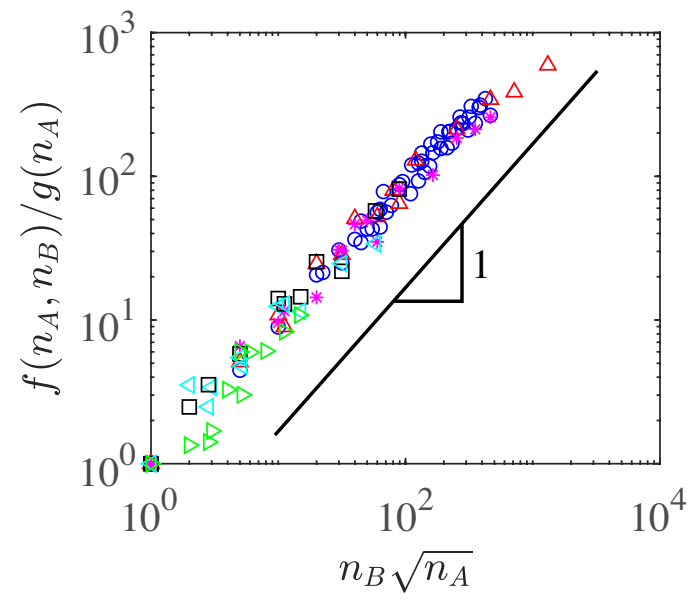

Fig. 5 Forces acting on a granular raft. Fitting coefficient of the speed of the granular raft $\mathrm{A}$, as a function of $n_{B} \sqrt{n_{A}}$, for different densities and sizes of particles (green right-pointing triangles: plastic beads, $\rho_{s}=1,420 \mathrm{~kg} \cdot \mathrm{m}^{-3}, R_{\text {part }}=2.5 \mathrm{~mm}$; blue left-pointing triangles: plastic beads, $\rho_{s}=1,420 \mathrm{~kg} \cdot \mathrm{m}^{-3}, R_{\text {part }}=2 \mathrm{~mm}$; black squares: ceramic beads, $\rho_{s}=4,800 \mathrm{~kg} \cdot \mathrm{m}^{-3}, R_{\text {part }}=0.45 \mathrm{~mm}$; pink stars: ceramic beads, $\rho_{s}=4,800 \mathrm{~kg} \cdot \mathrm{m}^{-3}, R_{\text {part }}=0.35 \mathrm{~mm}$; blue circles: ceramic beads, $\rho_{s}=3,800 \mathrm{~kg} \cdot \mathrm{m}^{-3}, R_{\text {part }}=0.45 \mathrm{~mm}$; red upward-pointing triangles: ceramic beads, $\rho_{s}=3,800 \mathrm{~kg} \cdot \mathrm{m}^{-3}, R_{\text {part }}=0.35 \mathrm{~mm}$ ). The solid line has a slope of 1.

version of equation (1):

$$
\left\{\begin{array}{l}
\nabla^{2} h=\frac{h}{\ell_{c}^{2}} \\
h(r \rightarrow \infty)=0 \\
h\left(r=R_{\text {raft }}\right)=h_{\text {raft }}
\end{array}\right.
$$

which gives us the following expression:

$$
\frac{d h}{d L}=\frac{h_{\text {raft }} / \ell_{c}}{K_{0}\left(R_{\text {raft }} / \ell_{c}\right)} K_{1}\left(\frac{L}{\ell_{c}}\right) .
$$

with $K_{0}$ the modified Bessel function of the second kind of order zero.

Equation (9) can be reconciled with the scaling $F_{\text {cap } A \rightarrow B} \propto n_{A}$ if we show that the modified aspect ratio of a raft $\frac{h_{\text {raft }} / \ell_{c}}{K_{0}\left(R_{\text {raft }} / \ell_{c}\right)}$ is linear with its number of particles $n$.

\subsection{Experimental and numerical description of a raft shape}

Experimentally, we measure $h_{\text {raft }}$ from side views of the granular raft (blue arrows, figure 6a). We then measure the area of the raft $A_{\text {raft }}$ from top views (see video 2 of the supplementary information) and calculate the equivalent radius by $R_{\text {raft }}=\sqrt{A_{\text {raft }} / \pi}$. The experimental modified aspect ratio is plotted in black diamonds in figure $6 \mathrm{~b}$ for different types of beads and raft sizes, as a function of the effective weight of a raft $F_{\text {vert }}$, that we define as the difference between the weight of the particles and their buoyancy:

$$
F_{\text {vert }}(n)=n F_{\text {vert }}(1)=n \frac{4}{3} \pi R_{\text {part }}^{3} g\left(\rho_{s}-\frac{\rho_{o}+\rho_{w}}{2}\right)
$$

with $n$ the number of particles in the raft. In equation (10), both the weight and the buoyancy of the particles are considered linear with $n$, with the supplementary assumption that a bead is equally immersed in oil and water. However, we are aware that the generalized buoyancy of the raft, which takes into account surface tension effect, is not strictly additive, as discussed in previous collective sinking experiments 14 . 
All the data collapse onto a single curve, and the modified aspect ratio appears linear with $F_{\text {vert }}$ (a power law regression on the experimental data gives an exponent of 1.01), which is by definition proportional to $n$. As a consequence, the modified aspect ratio is linear with the number of particles.

To this experimental evidence, we add a numerical confirmation for the morphology of a granular raft, using the code described in 21 . The raft is simulated as an axisymmetric continuous membrane of thickness $2 R_{\text {part }}$, with a given volume fraction of a material of density $\rho_{s}$, a complementary volume fraction of a material of density $\left(\rho_{o}+\rho_{w}\right) / 2$, and a tension $\vec{T}$ directed tangentially to the raft surface. As long as the tension remains positive, a static solution is found, and the shape of both the granular raft and the interface beyond can be solved numerically.

In figure 6, a, the simulated interface is plotted on top of experimental visualisations of the corresponding granular raft, with a visually good agreement for the top three photos. For the last two granular rafts shown in figure 6a, no numerical result is available: the simulations predict the sinking of the raft for such a deformation. This is probably due to the simplicity of the model used for the simulations, the limitations of which are discussed in our previous work 21 . As a consequence, the numerics can only give us information for part of the sizes accessible experimentally. However, they allow us to explore a far broader range of densities and radii of particles, and appear in that sense complementary to the experiments. The numerical measurement of the modified aspect ratio is represented in colored circles in figure 6. Here again, the data collapse onto the same curve when represented as a function of $F_{\text {vert }}$, over more than four decades, with an exponent of 1.08 for a power fitting law. In conclusion, we recover the following result both experimentally and numerically:

$$
\frac{h_{\text {raft }} / \ell_{c}}{K_{0}\left(R_{\text {raft }} / \ell_{c}\right)} \propto n
$$

Interestingly, in figure $6 \mathrm{~b}$, we find that beyond a critical vertical force, the linear relation between the modified aspect ratio and $F_{\text {vert }}$ no longer holds (see the right portion of the blue curve). Such a curve corresponds to a situation where the raft reaches a maximal depth which corresponds to a balance between the hydrostatic pressure and the raft weight, and then extends indefinitely as more particles are added 21 . As a consequence, $h_{\text {raft }}$ is bounded, leading to the saturation of the capillary force. Since $K_{0}\left(R_{\text {raft }} / \ell_{c}\right) \sim \sqrt{\frac{\pi}{2 R_{\text {raft }}}} e^{-R_{\text {raft }}}$ and $F_{\text {vert }} \propto R_{\text {raft }}^{2}$, it explains the vertical deviation in figure 6 b for large rafts.

Equation (11) can also be recovered theoretically in the limit of small deformations (see supplementary information for more details). All these results validate that:

$$
F_{\text {cap } B \rightarrow A}=n_{A} n_{B} F_{\text {cap } 1 \rightarrow 1}
$$

\subsection{The drag force on each raft}

Finally, we need to explain how the drag force depends on the raft geometry:

$$
F_{\text {drag } A}=\sqrt{n_{A}} F_{\text {drag } 1}
$$

One could understand the drag dependency with $n_{A}$ by considering the equivalent sphere of radius $R_{\text {raft }}$. For such a sphere, the Stokes drag is proportional to $R_{\text {raft }}$, which for a large range of granular raft sizes evolves as $\sqrt{n}$. This argument does not hold for the largest granular rafts (see for instance the last photo of figure 6), for which the high curvature of the raft makes $R_{\text {raft }}$ deviate from this law. However at first order, $R_{\text {raft }} \propto \sqrt{n}$, leading to the expected drag on the granular raft. The proportionality between the drag force and the raft radius is still quite surprising, since the shape of a granular raft is not a perfect sphere, but more a curved disk.

\section{Discussion}

A number of hypotheses underlying the approach described in this paper are no longer valid for a granular raft. They give us some clues to understand the limits of our model. In particular we observe an increasing discrepancy between the fit and the experimental data measured for the velocities when the rafts become large (the two highest velocities of figure 4. b for $n_{A}=20$ and 40). The two-sphere canonic example we use to derive equations (3) and (5) strongly relies on the assumption of small deformations around spherical beads (small in relation to the capillary length). With granular rafts, we can reach a vertical deformation of the interface up to almost half the capillary length. Moreover, the morphology of a raft, a two-dimensional circular monolayer of beads, cannot be approximated as a spherical shape, and one could therefore expect the drag to be drastically different. Finally, the viscous hypothesis used for the calculation of the drag can be questioned for the bigger rafts. In this case, the maximum speed reached during their motion is almost a hundred times higher than for two single beads, leading to a Reynolds number of order one. All these differences may change the amplitude of the forces through $f$ and $g$ and even the form of these functions.

We also assumed that the capillary force is proportional to $K_{1}\left(L / \ell_{c}\right)$, a result that comes from the linearized solution of equation (11). However, for some experiments, when two dense granular rafts interact, as in figure 7, equation (1) becomes highly non-linear, and the approximate linear solution is no longer sufficient to account for the curvature of the interface. Figure 7 illustrates this limitation of our model: on top of the experimental visualisation of the interface when two granular rafts are almost in contact, we plot the interfacial shape we would get if the deformations could be superimposed, deduced from the experimental measurement of the shape of the interface around a single granular raft of the same size. The superposition principle seems to account rather well for the curvature on the left and right side of each raft, but clearly overestimates the depth of the interface between the two rafts, leading to an error on the calculation of the capillary force. This could be part of the explanation for the disagreement between the fit and the measured speed in figure 4 for the motion of the two biggest rafts.

Another important feature that we did not take into account is the lateral extension of a granular raft. As already emphasized, the derivation of equation (2) for the capillary force between two spheres at an interface relies on various hypotheses, including the size of the particles: $R_{\text {part }} \ll \ell_{c}$. However, the largest rafts, as il- 

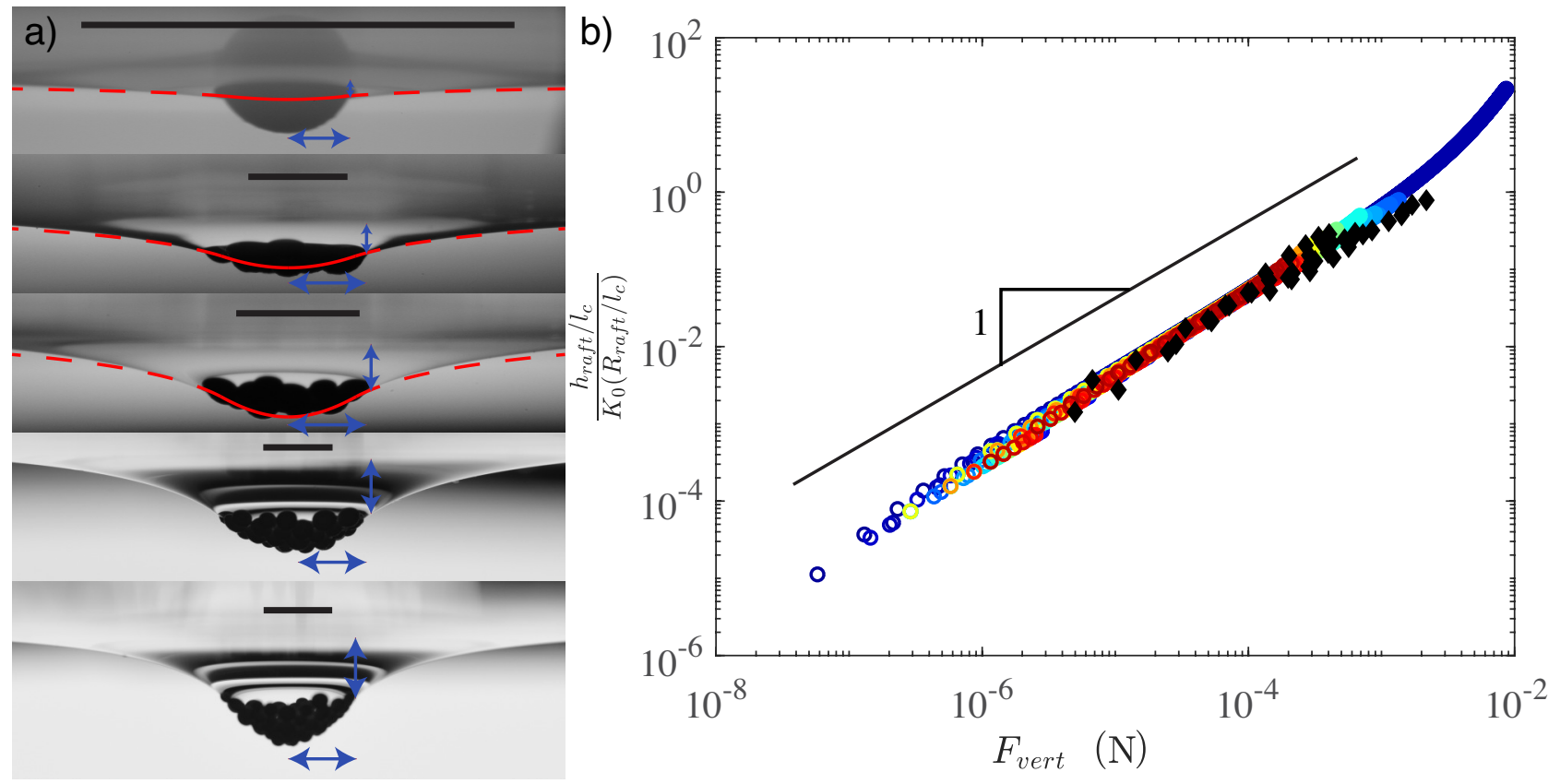

Fig. 6 Morphology and weight of a granular raft. Relation between the deformation of the interface and the weight of the granular raft, for a broad variety of densities, radii and numbers of particles. a) Side view of a granular raft for an increasing number of ceramic particles (density $\rho_{s}=4,800 \mathrm{~kg} \cdot \mathrm{m}^{-3}$, radius of a single particle $R_{\text {part }}=0.35 \mathrm{~mm}$ ). In the top three photos, the red line represents the shape of the interface calculated by the numerical simulation for a membrane of a radius similar to the background photo (dotted line: oil-water interface, solid line: granular raft). The blue arrows indicate the measurement of $R_{\text {raft }}$ and $h_{\text {raft }}$. Scale bars: $3 \mathrm{~mm}$ in each photo. b) Modified aspect ratio $\frac{h_{\text {raft }} / \ell_{c}}{K_{0}\left(R_{\text {raft }} / \ell_{c}\right)}$ as a function of the effective weight of the raft $F_{\text {vert }}$, for experiments (black diamonds), and numerical simulations (colored circles). The solid line has a slope of 1. The vertical errors bars for the experiments are of the order of the size of the individual points, and are thus not displayed for clarity reasons.

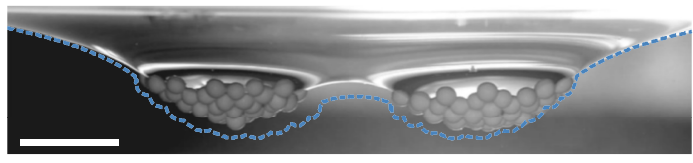

Fig. 7 Example of the failure of the linear superposition assumption. Comparison between a side view of two granular rafts during their motion towards one another (background photo) and the expected interfacial shape obtained if the assumption of small deformations was valid (blue dotted curve). Each of the rafts is made of 50 ceramic spherical beads (density $\rho_{s}=3,800 \mathrm{~kg} \cdot \mathrm{m}^{-3}$, radius of a single particle $R_{\text {part }}=0.45 \mathrm{~mm}$ ), in an axisymmetric configuration. The photo is taken $0.1 \mathrm{~s}$ before contact. Scale bar: $5 \mathrm{~mm}$.

lustrated in figure 7 for instance, can exhibit a diameter of the order of the capillary length. In this situation equation (2) should not be valid anymore. Here again, this could explain the discrepancy between our experimental data and the corresponding fitting curve for large rafts.

Despite all these limits, our model still accounts for the major part of our data, proving that in a first approximation, a granular raft does behave as a heavy membrane which is not deformed by the interaction with other rafts.

\section{Conclusion}

We have investigated the interaction dynamics of a two-body system formed after the clustering of beads into granular rafts at an oil-water interface. In particular we described the capillary forces generated by each granular raft, as well as the drag due to their individual motion. Because the deformation around a granular raft strongly depends on its size (depth and width), the capillary interaction can surpass by several orders of magnitude the forces created by individual beads, with a very strong dependence on the number of particles. A generalization of the two-particle model is found to account very satisfactorily for the motion of the granular rafts, and only starts to deviate from the experimental data for objects of dimensions close to the capillary length. Despite the clear non-linearity of the equations describing the shape of the interface for such large rafts, the capillary force appears linear both with the size of the attracting and the attracted raft. The drag, on the other hand, increases linearly with the radius of the considered raft (or in other terms, as the square root of the number of particles).

In this paper we have completely neglected the intrinsic discrete nature of a granular raft by modeling it as a twodimensional membrane. However, in some cases the granular aspect of the raft may play an important role. We have observed that rafts made of smaller particles (diameter $<400 \mu \mathrm{m}$ ) show a weaker cohesion between the grains within the raft, which may lead to erosion and internal motions for a fast enough raft displacement. Further work is thus required to characterize this phenomenon and investigate the role of shape changes on the overall interaction of granular objects at a liquid interface. Discrete aspects of the raft, as well as elasticity, could be also included in future developments of the model. 


\section{Conflicts of interest}

There are no conflicts to declare.

\section{Notes and references}

1 N. J. Mlot, C. A. Tovey and D. L. Hu, Proceedings of the National Academy of Sciences, 2011, 108, 7669-7673.

2 D. L. Hu and J. W. Bush, Nature, 2005, 437, 733.

3 U. Srinivasan, D. Liepmann and R. T. Howe, Journal of Microelectromechanical Systems, 2001, 10, 17-24.

4 T. D. Clark, J. Tien, D. C. Duffy, K. E. Paul and G. M. Whitesides, Journal of the American Chemical Society, 2001, 123, 7677-7682.

5 N. Bowden, A. Terfort, J. Carbeck and G. M. Whitesides, Science, 1997, 276, 233-235.

6 P. A. Kralchevsky and K. Nagayama, Advances in colloid and interface science, 2000, 85, 145-192.

7 K. D. Danov, P. A. Kralchevsky, B. N. Naydenov and G. Brenn, Journal of colloid and interface science, 2005, 287, 121-134.

8 K. D. Danov and P. A. Kralchevsky, Advances in colloid and interface science, 2010, 154, 91-103.

9 L. Botto, E. P. Lewandowski, M. Cavallaro and K. J. Stebe, Soft Matter, 2012, 8, 9957-9971.

10 D. Stamou, C. Duschl and D. Johannsmann, Physical Review E, 2000, 62, 5263.

11 I. B. Liu, N. Sharifi-Mood and K. J. Stebe, Annual Review of Condensed Matter Physics, 2018, 9, 283-305.

12 E. P. Lewandowski, J. A. Bernate, A. Tseng, P. C. Searson and K. J. Stebe, Soft Matter, 2009, 5, 886-890.

13 M. Cavallaro, L. Botto, E. P. Lewandowski, M. Wang and K. J. Stebe, Proceedings of the National Academy of Sciences, 2011,
108, 20923-20928.

14 D. Vella, Annual Review of Fluid Mechanics, 2015, 47, 115135.

15 M. Nicolson, Mathematical Proceedings of the Cambridge Philosophical Society, 1949, pp. 288-295.

16 D. Vella and L. Mahadevan, American journal of physics, 2005, 73, 817-825.

17 W. Gifford and L. Scriven, Chemical Engineering Science, 1971, 26, 287-297.

18 N. D. Vassileva, D. van den Ende, F. Mugele and J. Mellema, Langmuir, 2005, 21, 11190-11200.

19 M.-J. Dalbe, D. Cosic, M. Berhanu and A. Kudrolli, Physical Review E, 2011, 83, 051403.

20 M. Abkarian, S. Protière, J. M. Aristoff and H. A. Stone, Nature communications, 2013, 4, 1895.

21 S. Protière, C. Josserand, J. M. Aristoff, H. A. Stone and M. Abkarian, Physical review letters, 2017, 118, 108001.

22 N. Xue, S. Wu, S. Sun, D. QueİĄreİĄ and Q. Zheng, Langmuir, 2014, 30, 14712-14716.

23 J.-C. Loudet, A. M. Alsayed, J. Zhang and A. G. Yodh, Physical review letters, 2005, 94, 018301.

24 A. Dani, G. Keiser, M. Yeganeh and C. Maldarelli, Langmuir, 2015, 31, 13290-13302.

25 G. Batchelor, Journal of Fluid Mechanics, 1976, 74, 1-29.

26 P. A. Kralchevsky and K. Nagayama, Langmuir, 1994, 10, $23-$ 36.

27 V. Paunov, P. Kralchevsky, N. Denkov, I. Ivanov and K. Nagayama, Colloids and surfaces, 1992, 67, 119-138.

28 O. D. Velev, N. D. Denkov, V. N. Paunov, P. A. Kralchevsky and K. Nagayama, Langmuir, 1993, 9, 3702-3709. 